\title{
ALGEBRAS GENERATED BY TWO BOUNDED HOLOMORPHIC FUNCTIONS
}

\author{
MICHAEL I. STESSIN PASCAL J. THOMAS
}

\begin{abstract}
We study the closure in the Hardy space or the disk algebra of algebras generated by two bounded functions, of which one is a finite Blaschke product. We give necessary and sufficient conditions for density or finite codimension (of the closure) of such algebras. The conditions are expressed in terms of the inner part of some function which is explicitly derived from each pair of generators. Our results are based on identifying $z$-invariant subspaces included in the closure of the algebra.
\end{abstract}

\section{INTRODUCTION}

Let $g$ be an inner function in the unit disk. It is possible to prove that if $h \in H^{2}$ satisfies the condition $h\left(z_{1}\right)=h\left(z_{2}\right)$ for every pair $z_{1}, z_{2} \in \mathbb{D}$ such that $g\left(z_{1}\right)=g\left(z_{2}\right)$, then $h$ is in the $H^{2}$-closure of the algebra of polynomials in $g, \mathbb{C}[g]$, and vice versa (the proof rests on the fact that, in the case where $g(0)=0$, the self-mapping $g$ of the circle is Lebesgue measure-preserving). In fact, this result holds for a class of functions much broader than inner functions. It means that the $\mathrm{H}^{2}$-closure of $\mathbb{C}[g]$ is determined by the family of level sets of the function $g$. In particular, if $g$ is a Riemann mapping of $\mathbb{D}$ onto a polynomially convex domain $\Omega$ with a reasonable boundary, the algebra $\mathbb{C}[g]$ is dense in $H^{2}(\mathbb{D})$ (on this topic, the interest reader may consult [5]).

It is natural to ask if a similar result holds for algebras generated by two bounded functions. In particular, if a pair of functions $g_{1}, g_{2}$ separates points of the unit disk, under what condition is the algebra $\mathbb{C}\left[g_{1}, g_{2}\right]$ dense in $H^{2}$ ?

The case of closures of algebras generated by two disk-algebra functions (closure is taken in the disk-algebra metric) was intensively studied (cf [11], [1], [10]). Some conditions for the density of such an algebra were found (we will discuss some of those results in comparison with those obtained in the present paper in section 7 below).

In a special case when both $g_{1}$ and $g_{2}$ are polynomials the result similar to the one for algebras with one generator holds. Namely, the following Theorem follows directly from Proposition 1 of [3].

Theorem A. Let $p_{1}$ and $p_{2}$ be two polynomials such that $\left|p_{1}^{\prime}(z)\right|+\left|p_{2}^{\prime}(z)\right|>0$ for all $z \in \mathbb{C}$ and their degrees are mutually prime. Then the algebra $\overline{\mathbb{C}\left[p_{1}, p_{2}\right]}$ has finite codimension in $H^{2}$. If, in addition, $p_{1}$ and $p_{2}$ separate points of the unit disk, then $\mathbb{C}\left[p_{1}, p_{2}\right]$ is dense in $H^{2}$.

${ }^{0} 1991$ AMS subject classification: primary 30D55, secondary 46E25, 30D50

${ }^{0}$ The second-named author would like to thank the University at Albany, Harvard University, and Brown University for their hospitality during the completion of this work. 
The conditions that the derivatives of $p_{1}$ and $p_{2}$ do not vanish simultaneously and the degrees of $p_{1}$ and $p_{2}$ are mutually prime are too restrictive. As we will show below, they could be replaced with one much less restrictive condition.

If the generators are not polynomials, the condition of point separation is not sufficient for the density of the algebra in $H^{2}$. The following simple example illustrates this. Let $\chi(z)=\exp \left\{\frac{z+1}{z-1}\right\}$ be the singular inner function determined by the unit point mass at 1 , and $\psi(z)=z \chi(z)$. Clearly, $\chi$ and $\psi$ separate points of the unit disk. At the same time, every polynomial in $\chi$ and $\psi$ belongs to the subspace $L=\{1\}+\chi H^{2}$, where $\{1\}$ is the one-dimensional subspace of $H^{2}$ consisting of constants, and $\chi H^{2}$ is the $z$-invariant subspace generated by $\chi$. Since the codimension of $\overline{\chi H^{2}}$ is infinite, this implies that $\mathbb{C}[\chi, \psi]$ is not dense in $H^{2}$.

In this example both generators have non-trivial singular parts. What happens if one of them is analytic in the closed disk? The following argument pertains to the case when one of the generators is a Blaschke product of order two. It shows that even in this case the $H^{2}$ codimension of the closure of an algebra could be infinite, while its generators separate points of the unit disk. The study of this simple case also gives the flavor of the work which we carry out in the body of the paper, where we deal with the case where one generator is a Blaschke product of any finite order $n \geq 2$.

Let $|a|<1$ and $B(z)=c z \varphi(z)$, where $\varphi(z)=\frac{a-z}{1-\bar{a} z}$ is a Möbius involution of the disk, and $c$ a constant of modulus 1 . Given any $z \in \mathbb{D}$, there is at most another point $w$ of the disk such that $B(w)=B(z)$, given by $w=\varphi(z)$. There is exactly one $z_{0} \in \mathbb{D}$ such that $z_{0}=\varphi\left(z_{0}\right)$, and it is the only zero of $B^{\prime}$. In the simplest case where $B(z)=z^{2}, \varphi(z)=-z$ and $z_{0}=0$.

Any function $h \in H^{p}(\mathbb{D})$ which satisfies $h \circ \varphi(z)=h(z)$ can be rewritten $h=h_{1} \circ B$, where $h_{1}$ is well-defined from the set-theoretic point of view because of the hypothesis, is holomorphic outside of the critical point of $B$ by taking local inverses, and is actually holomorphic everywhere by removing the isolated singularity. If $p=\infty, h_{1}$ is clearly bounded. If $p<\infty$, since the mapping from the unit circle to itself induced by $B$ is $\mathcal{C}^{\infty}$ with nonvanishing derivative and 2-to-1, we have $h_{1} \in H^{p}(\mathbb{D})$. We then write $h \in H^{p}(B)$.

Since $B(0)=0$, the composition operator $C_{B}\left(C_{B}(f)=f \circ B\right)$ is an isometry of $H^{p}$ into $H^{p}$. Therefore, $\overline{\mathbb{C}[B]}=H^{p}(B)$, where $\mathbb{C}[B]$ stands for the polynomials in $B$, and the closure is taken either with respect to $H^{p}$ convergence when $p<\infty$, or to bounded pointwise convergence when $p=\infty$.

In particular, let $g \in H^{\infty}(\mathbb{D})$, then $g+g \circ \varphi$ and

$$
\Gamma(g)(z):=\frac{g(z)-g \circ \varphi(z)}{z-\varphi(z)}
$$

are in $H^{\infty}(B)$ (the possible singularity is easily seen to be removable). Furthermore,

$$
g-g \circ \varphi=2 g-(g+g \circ \varphi)
$$

shows that $g-g \circ \varphi \in \mathbb{C}\left[H^{\infty}(B), g\right] \subset \overline{\mathbb{C}[B, g]}$.

We claim that the following statement is true. 
Proposition. Let $0<p<\infty$ and $g \in H^{\infty}(\mathbb{D})$. When the closure is taken with respect to $H^{p}, \overline{\mathbb{C}[B, g]}=\overline{\mathbb{C}[B]}+I(g) H^{p}(\mathbb{D})=H^{p}(B)+I(g) H^{p}(\mathbb{D})$, where $I(g)$ is the inner factor in the canonical inner-outer factorization of $\Gamma(g)$.

The proof of this result is as follows. Let $h \in H^{p}(\mathbb{D})$. Then

$$
h(z) \Gamma(g)(z)=\frac{1}{2}(h(z)+h \circ \varphi(z)) \Gamma(g)(z)+\frac{1}{2} \frac{h(z)-h \circ \varphi(z)}{z-\varphi(z)}(g(z)-g \circ \varphi(z)) .
$$

By applying the above decomposition to the terms of a sequences of polynomials $h_{\nu}$ tending to $h$ in $H^{p}$, it is then easy to see that the first term is in $\overline{\mathbb{C}[B]} \subset \overline{\mathbb{C}[B, g]}$, while $\frac{h(z)-h \circ \varphi(z)}{z-\varphi(z)}$ is multiplied by $g-g \circ \varphi$, which has been show above to be in $\overline{\mathbb{C}[B, g]}$. This proves that $\Gamma(g) H^{p}(\mathbb{D}) \subset \overline{\mathbb{C}[B, g]}$, and $\overline{\Gamma(g) H^{p}(\mathbb{D})}=I(g) H^{p}(\mathbb{D})$, by Beurling's theorem.

Conversely, consider a monomial of the form $M=g^{\alpha} B^{\beta}$. If $\alpha=0$, then $M \in \mathbb{C}[B]$. If $\alpha \geq 1$,

$$
\begin{aligned}
& g^{\alpha}=\left(\frac{1}{2}(g-g \circ \varphi)+\frac{1}{2}(g+g \circ \varphi)\right)^{\alpha} \\
& =\frac{1}{2^{\alpha}} \sum_{l=0}^{\alpha}\left(\begin{array}{c}
\alpha \\
l
\end{array}\right)(g-g \circ \varphi)^{l}(g+g \circ \varphi)^{\alpha-l} \in H^{\infty}(B)+(g-g \circ \varphi) H^{\infty}(B),
\end{aligned}
$$

as can be seen by splitting the last sums into terms with $l$ even or odd respectively. Now $g-g \circ \varphi=(z-\varphi) \Gamma(g) \in \Gamma(g) H^{\infty}(\mathbb{D})$.

This proves that $\mathbb{C}[B, g] \subset \mathbb{C}[B]+I(g) H^{p}(\mathbb{D})$. To prove the corresponding inclusion for the $H^{p}$ closures, it will be enough to show that $\overline{\mathbb{C}[B]}+I(g) H^{p}(\mathbb{D})$ is closed in $H^{p}$.

Each term in the sum is a closed subspace. For any $f \in H^{p}(\mathbb{D})$, write

$$
f=\pi_{1}(f)+\pi_{2}(f):=\frac{1}{2}(f+f \circ \varphi)+\frac{1}{2}(f-f \circ \varphi) .
$$

Then the maps $\pi_{1}$ and $\pi_{2}$ are continuous from $H^{p}(\mathbb{D})$ to itself, $\pi_{1}$ is a projection onto $\overline{\mathbb{C}[B]}=H^{p}(B)$, and $H^{p}(B) \subset \operatorname{Ker} \pi_{2}$. Suppose that $f=\lim _{n}\left(x_{n}+y_{n}\right)$, with $x_{n} \in H^{p}(B)$ and $y_{n} \in I(g) H^{p}(\mathbb{D})$. Then

$$
\pi_{2}(f)=\lim _{n \rightarrow \infty}\left(\pi_{2}\left(x_{n}\right)+\pi_{2}\left(y_{n}\right)\right)=\lim _{n \rightarrow \infty} \pi_{2}\left(y_{n}\right),
$$

which proves the existence of the last limit. Since $\Gamma(g) \circ \varphi=\Gamma(g)$, we have $I(g) \circ \varphi=$ $I(g)$, and if $y_{n}=I(g) w_{n}$, with $w_{n} \in H^{p}(\mathbb{D})$, we have

$$
\pi_{2}\left(y_{n}\right)=I(g)\left(w_{n}-w_{n} \circ \varphi\right) \in I(g) H^{p}(\mathbb{D}),
$$

therefore $\pi_{2}(f) \in I(g) H^{p}(\mathbb{D})$ and $f \in \overline{\mathbb{C}[B]}+I(g) H^{p}(\mathbb{D})$, q.e.d.

Corollary. $\mathbb{C}[B, g]$ is dense in $H^{p}$ if and only if $\Gamma(g)$ is an outer function.

In the case where $B(z)=z^{2}$ and $\Gamma(g)$ is not an outer function, it is easy to find an annihilator for $\overline{\mathbb{C}[B]}+I(g) H^{p}(\mathbb{D})$ directly. Here $\varphi(z)=-z$. By Beurling's theorem, there exists $h \in H^{q}(\mathbb{D})$ such that $\int_{\partial \mathbb{D}} h \bar{f}=0$, for any $f \in I(g) H^{p}(\mathbb{D})$. We claim $h_{1}:=h-h \circ \varphi$ is the annihilator we are looking for. Indeed, for any $f \in I(g) H^{p}(\mathbb{D})$,

$$
\int_{\partial \mathbb{D}}(h \circ \varphi) \bar{f}=\int_{\partial \mathbb{D}} h \overline{f \circ \varphi}=0,
$$


since $f \circ \varphi \in I(g) H^{p}(\mathbb{D})$. So $\int_{\partial \mathbb{D}} h_{1} \bar{f}=0$.

Furthermore, if $f \in H^{p}(B)$, then $f \circ \varphi=f$ and

$$
\int_{\partial \mathbb{D}}(h-h \circ \varphi) \bar{f}=\int_{\partial \mathbb{D}} h \bar{f}-\int_{\partial \mathbb{D}}(h \circ \varphi) \bar{f}=\int_{\partial \mathbb{D}} h \bar{f}-\int_{\partial \mathbb{D}} h \overline{f \circ \varphi}=0 .
$$

The case of a general Blaschke product $B$ of degree 2 can be reduced (as far questions of density and the computation of $I(g)$ are concerned) to the case of $z^{2}$ by using a Möbius map from the disk to itself taking the unique critical point of $B$ to 0 . We omit the details.

If $B(z)=z^{2}$ and $g(z)=\chi(z)=\exp \frac{z+1}{z-1}$ as above, then a computation shows that $\Gamma(\chi)$ has no zeroes, and $\chi$ is continuous up to the boundary and non zero for any $\zeta \in \partial \mathbb{D} \backslash\{1\}$. It is then easy to see that $\Gamma(\chi)$ is continuous on $\partial \mathbb{D} \backslash\{+1,-1\}$, so that any singular inner factor of $\Gamma(\chi)$ would have to have its singular measure concentrated on those two points; but $\Gamma(\chi)$ admits a finite non-zero radial limit at each of those points, which precludes any singular inner factor. Thus, $\mathbb{C}\left[z^{2}, \chi(z)\right]$ is dense in $H^{2}$.

On the other hand, still for $B(z)=z^{2}$, if we pick $g(z)=z \chi\left(z^{2}\right)$, then $\Gamma(g)=\chi\left(z^{2}\right)$, which is singular inner. Thus, $\mathbb{C}[B, g]$ is not dense in $H^{2}$. At the same time $z^{2}$ and $g(z)$ obviously separate points of $\mathbb{D}$ (which follows also from the fact that $\Gamma(g)$ does not vanish inside $\mathbb{D}$ ). Moreover, by the above Proposition, for every function $f \in H^{2}$ which is orthogonal to the $z$-invariant subspace $\chi H^{2}(\mathbb{D})$ the function $h(z)=z f\left(z^{2}\right)$ is orthogonal to $\mathbb{C}[B, g]$, so $\overline{\mathbb{C}[B, g]}$ has infinite codimension in $H^{2}$.

\section{Notations and statements of results}

In this paper we study algebras with two generators, one of which is a finite Blaschke product of degree $n$ :

$$
B(z):=\prod_{j=1}^{n} \frac{a_{j}-z}{1-\bar{a}_{j} z}
$$

where $a_{1}, \ldots, a_{n} \in \mathbb{D}$. Given a point $z \in \mathbb{D}$, there are exactly $n$ points in the unit disk (counting multiplicities), $z:=\varphi_{0}(z), \varphi_{1}(z), \ldots, \varphi_{n-1}(z)$ such that $B\left(\varphi_{j}(z)\right)=$ $B(z), j=1, \ldots, n-1$. For $f \in H^{\infty}$ we define $\Gamma_{B}(f)$ by

$$
\Gamma_{B}(f)(z)=\frac{\prod_{j=1}^{n-1}\left(f(z)-f \circ \varphi_{j}(z)\right)}{\prod_{j=1}^{n-1}\left(z-\varphi_{j}(z)\right)} .
$$

In what follows we usualy omit the subscript for $\Gamma_{B}$ and write $\Gamma(f)$ instead of $\Gamma_{B}(f)$. It will be shown in section 2 that for all $f \in H^{\infty}, \Gamma(f)$ is analytic and, since it is obviously bounded, is an $H^{\infty}$ function. Moreover, for all $0<p \leq \infty$ the mapping $f \longmapsto \Gamma(f)$ can be extended as a continuous mapping from $H^{p}$ to $H^{p /(n-1)}$.

The main result of this paper is the following Theorem.

Theorem B. Let $\mathcal{A}$ stand for the algebra of functions $\mathbb{C}[B, g]$ where $g \in H^{\infty}$. The closure of $\mathcal{A}$, denoted $\overline{\mathcal{A}}$, is taken with respect to the $H^{p}$ norm when $p<\infty$, and with respect to bounded pointwise convergence when $p=\infty$.

(1) For every $p>0$ the closure of $\mathcal{A}$ in $H^{p}$ contains the z-invariant subspace $\Gamma(g) H^{p}$.

(2) $\overline{\mathcal{A}}=H^{p}$ if and only if $\Gamma(g)$ is outer. 
(3) $\overline{\mathcal{A}}$ has finite codimension in $H^{p}$ if and only if the inner part of $\Gamma(g)$ is a finite Blaschke product.

As a corollary to the proof of Theorem B we obtain the following generalization of Theorem A (though $p_{1}$ is not a Blaschke product, $\Gamma_{p_{1}}\left(p_{2}\right)$ below can be defined in a similar way. The details may be found in section 4).

Theorem C. Let $p_{1}$ and $p_{2}$ be two polynomials. If $\Gamma_{p_{1}}\left(p_{2}\right)$ is not identically equal to zero, then the algebra $\overline{\mathbb{C}\left[p_{1}, p_{2}\right]}$ has finite codimension in $H^{2}$. Furthermore, $\mathbb{C}\left[p_{1}, p_{2}\right]$ is dense in $H^{2}$ if and only if $\Gamma_{p_{1}}\left(p_{2}\right)$ does not vanish in the open unit disk.

In the case when $\Gamma(g) \equiv 0$, Theorem B (as well as Theorem $\mathrm{C}$ ) does not give much information about the size of the closure of the algebra. It is shown in section 5 below that the identical vanishing of $\Gamma(g)$ implies a strong functional relation between $B$ and $g$. The following Theorem gives a necessary and sufficient condition for the identical vanishing of $\Gamma(g)$.

Theorem D. The function $\Gamma(g)$ vanishes identically in the unit disk if and only if there exist a function $\varphi$ from the disk to itself and a discrete subset $A$ of the disk such that for any $z \in \mathbb{D} \backslash A, \# \varphi^{-1}(z)>1$, such that $B=B_{1} \circ \varphi$ and $g=g_{1} \circ \varphi$, where $B_{1}$ and $g_{1}$ are functions defined on the disk.

A direct corollary to this result is

Theorem $\mathbf{D}^{\prime}$. The function $\Gamma(g)$ vanishes identically if and only if the closure of $\mathbb{C}[B, g]$ does not contain a non-trivial z-invariant subspace.

As another corollary to Theorem D we obtain the following result.

Corollary. If the order of the Blaschke product $B$ is a prime number, then, whenever the bounded analytic function $g$ is not in $H^{\infty}[B], \Gamma(g)$ does not vanish identicaly.

Finally, though the disk algebra $A(\overline{\mathbb{D}})$ is not our prime focus, our methods yield similar disk algebra results. It is easily seen that if $g$ is a disk algebra function, $\Gamma(g)$ is also in the disk algebra. The following Theorem holds.

Theorem E.

(1) Let $g \in A(\overline{\mathbb{D}})$. Then the closure of $\mathbb{C}[B, g]$ in $A(\overline{\mathbb{D}})$ contains $\Gamma(g) A(\overline{\mathbb{D}})$.

(2) $\mathbb{C}[B, g]$ is dense in $A(\overline{\mathbb{D}})$ if and only if $\Gamma(g)$ has no zeros in the closed unit disk.

(3) $\overline{\mathbb{C}[B, g]}$ has finite codimension in $A(\overline{\mathbb{D}})$ if and only if $\Gamma(g)$ has no singular inner factor and only a finite number of zeros in the closed unit disk.

The structure of this paper is as follows. Section 2 is devoted to some auxiliary results related to level sets of the Blaschke product $B$. Here we introduce the function $\Gamma(g)$ and prove its analyticity. In section 3 we prove the "necessary" part of Theorem 1. In section 4 we show how Theorem $\mathrm{C}$ follows from Theorem B. In this section we also give an estimate of the number of generators of a closed submodule of $H^{2}$ over a polynomial algebra expressed in terms of the function $\Gamma$. Section 5 deals with the case when $\Gamma(g) \equiv 0$. Theorems $\mathrm{D}$ and $\mathrm{D}^{\prime}$ are proved in this section. In section 6 we discuss $z$-invariant subspaces contained in the algebra's closure. Section 7 is devoted 
to applications to the disk algebra case. Here we also compare our results with previous ones obtained by J. Wermer and R. G. Blumenthal without the assumption that one of the generators be a finite Blaschke product. Finally, in section 8 we conclude the proofs of Theorems B and E.

Acknowledgements. The authors would like to thank John Wermer for stimulating discussions and the references [4], [12], and Emma Previato for pointing out and explaining [2]. We thank the referee for his careful comments and corrections.

\section{Auxiliary Results}

2.1. Level sets of $B$. For any $z \in \overline{\mathbb{D}}$, the set $B^{-1}(B(z))$ can be written as $\{z=$ : $\left.\varphi_{0}(z), \varphi_{1}(z), \ldots, \varphi_{n-1}(z)\right\}$, which are the $n$ solutions (possibly equal) of a polynomial equation of degree $n$; the maps $\varphi_{j}$ may be chosen holomorphic in $z$ near any point $z^{0}$ such that $\varphi_{j}\left(z^{0}\right)$ is a simple zero of $B-B\left(z^{0}\right)$ (equivalently, such that $B^{\prime}\left(z^{0}\right) \neq 0$ ), but cannot be chosen to be globally continuous.

Notice in particular that $B^{\prime}$ cannot vanish on the boundary $\partial \mathbb{D}$ of the disc, and that, since they all map the circle into itself and never coincide, the maps $\left(\varphi_{j}, 1 \leq j \leq\right.$ $n-1)$ can be ordered by circular order, and thus are all individually well-defined in a neighborhood of the unit circle. Explicitly, we can write $\varphi_{j}(\theta)=\exp \left(i \alpha_{j}(\theta)\right)$, where $\theta<\alpha_{j}(\theta)<\theta+2 \pi$. A priori, those choices only hold locally. Then label the $n-1$ distinct points of the unit circle $\left\{\varphi_{1}(1), \ldots, \varphi_{n-1}(1)\right\}$ by demanding $\alpha_{j}(0)<\alpha_{j+1}(0)$, $1 \leq j \leq n-2$. Because $B^{\prime}\left(e^{i \theta}\right) \neq 0$ for any $\theta$, we never have $\lim _{\theta \rightarrow \theta_{0}} \alpha_{j}(\theta)=$ $\lim _{\theta \rightarrow \theta_{0}} \alpha_{j+1}(\theta)$, so the set

$$
U_{j}:=\left\{\theta \in[0,2 \pi): \alpha_{j}, \alpha_{j+1} \in \mathcal{C}^{0}[0, \theta] \text { and } \forall \theta^{\prime} \in[0, \theta], \alpha_{j}(\theta)<\alpha_{j+1}(\theta)\right\}
$$

is both open and closed, so is equal to the whole interval $[0,2 \pi)$ and passing to the limit and using $B^{\prime}\left(e^{i \theta}\right) \neq 0$ again, we will have

$$
2 \pi<\alpha_{j}(2 \pi)<\alpha_{j+1}(2 \pi)<4 \pi, 1 \leq j \leq n-2,
$$

and therefore $\alpha_{j}(2 \pi)=\alpha_{j}(0)+2 \pi, 0 \leq j \leq n-1$, and we have liftings of continuous maps.

Furthermore, the quantities $\left|\varphi_{j}\left(e^{i \theta}\right)-\varphi_{k}\left(e^{i \theta}\right)\right|, 0 \leq \theta \leq 2 \pi, 0 \leq j \neq k \leq n-1$, are uniformly bounded away from 0 .

Near a multiple point $z^{0}$, so that $B-B\left(z_{0}\right)$ has a zero of order $N$, the $\varphi_{j}$ can be expressed as series in $\left(z-z^{0}\right)^{1 / N}$ (so they are actually continuous at the point $z^{0}$, if not in any neighborhood of it) (cf. for example [7]).

Symmetric polynomial (resp. rational) functions of $\varphi_{j}(z), 0 \leq j \leq n-1$ are welldefined and holomorphic (resp. meromorphic) on the whole disc. For a multiple point $z^{0}$, if $A \subset\{0, \ldots, n-1\}$ is such that $\varphi_{j}\left(z^{0}\right)=\varphi_{k}\left(z^{0}\right)$ for any $j, k \in A$, and $\varphi_{j}\left(z^{0}\right) \neq \varphi_{k}\left(z^{0}\right)$ for any $j \in A, k \notin A$, then the symmetric polynomial functions of $\left(\varphi_{j}(z), j \in A\right)$ are well-defined and holomorphic in some neighborhood of $z^{0}$ (as can be seen by factoring the equation).

In the special case that is of interest to us, since $\varphi_{0}(z)=z$ is always globally welldefined, the same is true of the symmetric functions of $\left(\varphi_{j}(z), 1 \leq j \leq n-1\right)$. More precisely, we have the following easy lemma. From now on, we shall always assume that $B$ vanishes at the origin, so in (1) we have $a_{1}=0$. This is no loss of generality as it can be obtained by composing our functions by a Möbius map of the disk. 
Lemma 1. Any symmetric polynomial (resp. rational function, resp. holomorphic function in the disk) in $\varphi_{j}, 0 \leq j \leq n-1$, can be written as a polynomial (resp. rational function) in $B(z)$.

Any symmetric polynomial (resp. rational function) in $\varphi_{j}, 1 \leq j \leq n-1$, can be written as a polynomial (resp. rational function, resp. holomorphic function in the disk) in $z$ and $B(z)$.

Proof. Denote the elementary symmetric functions in $n(\operatorname{resp} n-1)$ indeterminates by

$$
\begin{aligned}
\sigma_{k}\left(X_{0}, \ldots X_{n-1}\right) & :=\sum_{A \subset\{0, \ldots, n-1\}, \# A=k} \prod_{j \in A} X_{j}, \\
\text { resp. } \sigma_{k}^{\prime}\left(X_{1}, \ldots X_{n-1}\right) & :=\sum_{A \subset\{1, \ldots, n-1\}, \# A=k} \prod_{j \in A} X_{j} .
\end{aligned}
$$

It is clear that the $\sigma_{k}\left(\varphi_{0}(z), \ldots, \varphi_{n-1}(z)\right)$ depend only on $B(z)$. The polynomial equation which admits $\left\{\varphi_{0}(z), \ldots, \varphi_{n-1}(z)\right\}$ as solutions is

$$
\prod_{j=1}^{n}\left(a_{j}-X\right)=B(z) \prod_{j=1}^{n}\left(1-\bar{a}_{j} X\right) .
$$

Since $a_{1}=0$, the coefficient of $X^{n}$ is exactly $(-1)^{n}$, and all the coefficients of $X^{m}$, $0 \leq m \leq n$, are affine functions of $B(z)$, which proves the first assertion.

To prove the second assertion of the lemma, it will be enough to show that the $\sigma_{k}^{\prime}$ are polynomials in $X_{0}$ and $\sigma_{k}$. But

$$
\sigma_{k}\left(X_{0}, \ldots X_{n-1}\right)=X_{0} \sigma_{k-1}^{\prime}\left(X_{1}, \ldots X_{n-1}\right)+\sigma_{k}^{\prime}\left(X_{1}, \ldots X_{n-1}\right),
$$

with $\sigma_{0}=\sigma_{0}^{\prime}=1$, so the relation $\sigma_{k}^{\prime}=\sigma_{k}-X_{0} \sigma_{k-1}^{\prime}$ allows us to conclude by induction.

2.2. Auxiliary functions. For any $f \in \mathcal{O}(\mathbb{D})$, define

$$
\mathcal{D}(f)(z):=\prod_{j=1}^{n-1}\left(f(z)-f \circ \varphi_{j}(z)\right) .
$$

This is symmetric in $\left(\varphi_{j}(z), 1 \leq j \leq n-1\right)$, and, therefore, by Lemma 1 is globally holomorphic in $z$. In particular, $\mathcal{D}(z):=\prod_{j=1}^{n-1}\left(z-\varphi_{j}(z)\right)$ is a function which vanishes at $z^{0}$ if and only if $B^{\prime}\left(z^{0}\right)=0$ (it can be seen to be a discriminant).

Define $\Gamma(f)(z):=\mathcal{D}(f)(z) / \mathcal{D}(z)$. This is clearly meromorphic in the disc, but actually each singularity is removable. Indeed, near any point $z^{0}$ where for some $j$, $\varphi_{j}\left(z^{0}\right)=z^{0}$, we have

$$
f(z)=\sum_{m \geq 0} \alpha_{m}\left(z-z^{0}\right)^{m}, \quad f\left(\varphi_{j}(z)\right)=\sum_{m \geq 0} \alpha_{m}\left(\varphi_{j}(z)-z^{0}\right)^{m},
$$

where the second series converges in a neighborhood of $z^{0}$ since $\left|\varphi_{j}(z)-z^{0}\right|$ $=\left|\varphi_{j}(z)-\varphi_{j}\left(z^{0}\right)\right| \leq C\left|z-z^{0}\right|^{1 / N}$. Therefore,

$$
\begin{gathered}
f(z)-f\left(\varphi_{j}(z)\right)= \\
\left(z-\varphi_{j}(z)\right) \sum_{m \geq 1} \alpha_{m}\left(\left(z-z^{0}\right)^{m-1}+\left(z-z^{0}\right)^{m-2}\left(\varphi_{j}(z)-z^{0}\right)+\cdots+\left(\varphi_{j}(z)-z^{0}\right)^{m-1}\right),
\end{gathered}
$$


and the infinite series converges in a neighborhood of $z^{0}$ because its general term is bounded by $C m\left|\alpha_{m}\right|\left|z-z^{0}\right|^{(m-1) / N}$. Let $A:=\left\{j \geq 1: \varphi_{j}\left(z^{0}\right)=z^{0}\right\}$, then

$\prod_{j \notin A}\left(f(z)-f \circ \varphi_{j}(z)\right) /\left(z-\varphi_{j}(z)\right)$ is clearly holomorphic in a neighborhood of $z^{0}$, and

$$
\begin{aligned}
& \prod_{j \in A} \frac{\left(f(z)-f \circ \varphi_{j}(z)\right)}{\left(z-\varphi_{j}(z)\right)} \\
& =\prod_{j \in A} \sum_{m \geq 1} \alpha_{m}\left(\left(z-z^{0}\right)^{m-1}+\left(z-z^{0}\right)^{m-2}\left(\varphi_{j}(z)-z^{0}\right)+\cdots+\left(\varphi_{j}(z)-z^{0}\right)^{m-1}\right) .
\end{aligned}
$$

The coefficients of the product series are easily seen to be symmetric in $\left(\varphi_{j}(z), j \in A\right)$, therefore well-defined and holomorphic in a neighborhood of $z^{0}$, so the product on the left hand side of the equation, and therefore the function $\Gamma(f)$, has a removable singularity at $z^{0}$, as required.

The zeros of the function $\Gamma(f)$ have a simple interpretation in terms of self-intersection or cusps of the analytic graph $\{(B(\zeta), f(\zeta)), \zeta \in \mathbb{D}\} \subset \mathbb{C}^{2}$.

Lemma 2. Let $z \in \mathbb{D}$, then $\Gamma(f)(z)=0$ if and only if one of the following occurs:

(i) There exists $z^{\prime} \neq z$ such that $B\left(z^{\prime}\right)=B(z)$ and $f\left(z^{\prime}\right)=f(z)$;

(ii) $B^{\prime}(z)=f^{\prime}(z)=0$.

Proof. The product defining $\Gamma(f)$ vanishes if and only if one its factors does, i.e. there exists $j, 1 \leq j \leq n-1$, such that

$$
\frac{f(z)-f \circ \varphi_{j}(z)}{\left(z-\varphi_{j}(z)\right)}=0 .
$$

If $z \neq \varphi_{j}(z)$, this simply means that $f(z)=f\left(\varphi_{j}(z)\right)$, and we have case (i). If $z=\varphi_{j}(z), B$ is not locally invertible at $z$, therefore $B^{\prime}(z)=0$, and the vanishing of the factor means that

$$
0=\lim _{z^{\prime} \rightarrow z} \frac{f\left(z^{\prime}\right)-f \circ \varphi_{j}\left(z^{\prime}\right)}{\left(z^{\prime}-\varphi_{j}\left(z^{\prime}\right)\right)}=\lim _{z^{\prime} \rightarrow z} \int_{0}^{1} f^{\prime}\left(t z^{\prime}+(1-t) \varphi_{j}\left(z^{\prime}\right)\right) d t
$$

which happens exactly when $f^{\prime}(z)=0$.

\section{NECESSARY CONDITIONS FOR DENSITY OR FINITE CODIMENSION}

We shall now see how the function $\Gamma(g)$ defined above relates to the properties of the algebra $\mathbb{C}[B, g]$. The main result of this section is Theorem 5 .

Lemma 3. For $0<p \leq \infty$, the mapping defined by $f \mapsto \Gamma(f)$ is continuous from $H^{p}$ to $H^{p /(n-1)}$.

Proof. We have seen that $\Gamma(f)$ is holomorphic on the whole disc. By the remarks at the beginning of $\$ 2.1$, in a neighborhood of the unit circle the maps $\varphi_{j}$ are all globally defined, holomorphic, with a non-vanishing derivative, it is clear that each function $f \circ \varphi_{j}$ is an $H^{p}$ function : holomorphic on a neighborhood of the unit circle in $\mathbb{D}$, bounded for $p=\infty$, and for $p<\infty, f \circ \varphi_{j}(r \zeta)$ tends to $f \circ \varphi_{j}(\zeta)$ in the $L^{p}$ norm on the unit circle. The $H^{p}$-norm of $f \circ \varphi_{j}$ is bounded by $C\|f\|_{H^{p}}=C\|f\|_{L^{p}(\partial \mathbb{D})}$.

Since $\left|z-\varphi_{j}(z)\right|$ is bounded from below in a neighborhood of the unit circle, a similar $H^{p}$ bound holds for $\left(f-f \circ \varphi_{j}\right) /\left(z-\varphi_{j}(z)\right)$. Now the map $\Gamma$, seen as a map 
from $H^{p}$ to $L^{p /(n-1)}(\partial \mathbb{D})$, is a composition of linear and $(n-1)$-linear maps, so to check that it is continuous it is enough to see that

$$
\|\Gamma(f)\|_{L^{p /(n-1)(\partial \mathbb{D})}} \leq C\|f\|_{p}^{n-1},
$$

which follows from the above estimates and repeated use of Hölder's inequality.

For $p=\infty$, the required estimate follows immediately from $\left|z-\varphi_{j}(z)\right|$ being bounded from below.

Proposition 4. Let $\mathcal{A}:=\mathbb{C}[B, g]$, and $\overline{\mathcal{A}}$ be the closure of $\mathcal{A}$ with respect to the $H^{p}$ norm, for some $p \in(0, \infty)$. Let $\Gamma(g)=B_{g} S_{g} F_{g}$ be the canonical factorization of $\Gamma(g)$ into, respectively, Blaschke product, singular inner factor, and outer factor. Then for any $f \in \overline{\mathcal{A}}, \Gamma(f) \in B_{g} S_{g} H^{p /(n-1)}$.

Proof. Suppose first that $f \in \mathcal{A}$. Then, using the fact that $B \circ \varphi_{j}=B$ for any $j$,

$$
\begin{aligned}
f= & \sum_{\alpha, \beta \geq 0} a_{\alpha \beta} g^{\alpha} B^{\beta} \text { implies } \\
& \Gamma(f)=\Gamma(g) \prod_{j=1}^{n-1}\left(\sum_{\alpha \geq 1, \beta \geq 0} a_{\alpha \beta} B^{\beta}\left[\sum_{l=0}^{\alpha-1} g^{\alpha-1-l}\left(g \circ \varphi_{j}\right)^{l}\right]\right),
\end{aligned}
$$

so $\Gamma(f) \in \Gamma(g) \mathbb{C}\left[H^{\infty}(B), g\right]$, because the last product involves a finite set of powers of $B$ and polynomials which are symmetric in the $\left\{g \circ \varphi_{j}, 1 \leq n-1\right\}$, and therefore can be expressed as polynomials of $g$ and bounded holomorphic functions symmetric in $\left\{g \circ \varphi_{j}, 0 \leq n-1\right\}$, which are then necessarily bounded holomorphic functions of $B$.

Suppose $\mathcal{A} \ni f_{\nu} \rightarrow f$ in $H^{p}$. By the above we have $\Gamma\left(f_{\nu}\right)=\Gamma(g) q_{\nu}, q_{\nu} \in$ $\mathbb{C}\left[H^{\infty}(B), g\right]$. Now in the canonical factorization, the harmonic function $\log \left|F_{g}\right|$ is the Poisson integral of its boundary values, while $\log \left|S_{g}\right|$ is the Poisson integral of a singular measure $-\sigma_{g}$, where $\sigma_{g}$ is a positive measure (carried on a Borel set of Lebesgue measure 0$)$. Factoring both $\Gamma\left(f_{\nu}\right)$ and $q_{\nu}$, and using the essential uniqueness of the factorization, we see that

$$
\sigma_{f_{\nu}} \geq \sigma_{g}
$$

for all indices $\nu$ (their difference is a positive measure). Since $\Gamma\left(f_{\nu}\right)$ must converge to $\Gamma(f)$ in $H^{p /(n-1)}, \sigma_{f_{\nu}}$ converges weakly to $\sigma_{f}$, so $\sigma_{f} \geq \sigma_{g}$, which implies that $S_{g}$ divides $\Gamma(f)$.

On the other hand, $H^{p /(n-1)}$ convergence implies convergence of the function and all its derivatives uniformly on any compactum, and therefore since each $\Gamma\left(f_{\nu}\right)$ has zeroes at the points of $B_{g}^{-1}(0)$ with at least the same multiplicity, the same holds true for $\Gamma(f)$. Since dividing by $B_{g} S_{g}$ only affects the boundary values of $\log |\Gamma(f)|$ on a set of measure 0 , the remaining function is still in $H^{p /(n-1)}$, q.e.d.

Theorem 5. (1) If $\mathcal{A}$ is dense in $H^{p}$, then $\Gamma(g)$ is an outer function.

(2) If $\overline{\mathcal{A}}$ has finite codimension in $H^{p}$, then the inner part of $\Gamma(g)$ is a finite Blaschke product (and in particular there is no singular inner factor).

Proof. If $z \in \overline{\mathcal{A}}$, then by Proposition $甘, 1=\Gamma(z) \in B_{g} S_{g} H^{p /(n-1)}$, which implies that $\Gamma(g)$ has no inner factor by Beurling's theorem.

If $\overline{\mathcal{A}}$ has finite codimension $m$, then it must contain a non-zero polynomial $P$ of degree $m$. Then $\Gamma(P)$ is analytic across the unit circle, so cannot admit a singular 
inner factor. On the other hand, any zero $a$ of $\Gamma(g)$ in $\mathbb{D}$ provides a continuous functional on $H^{p}$ which vanishes on $\overline{\mathcal{A}}$ : in the case (i) of Lemma 2 , the functional is $f \mapsto f(a)-f\left(a^{\prime}\right)$, in the case (ii), $f \mapsto f^{\prime}(a)$. It is easy to see that if the set of zeros of $\Gamma(g)$ is infinite, this family of functionals is not of finite dimension, which contradicts the hypothesis.

\section{MAin RESUlts: SUFFICIENT CONDITIONS FOR DENSITY, GENERATING SETS FOR SUBMODULES}

Theorem 6. For any $p>0, \overline{\mathcal{A}}$ contains $\Gamma(g) H^{p}$.

Let us defer the proof of Theorem 6 to Section 8 in favor of a few observations.

First, by Beurling's theorem again, if $\Gamma(g)$ is outer, then the closure of $\Gamma(g) H^{p}$ is $H^{p}$ itself; and if the inner factor of $\Gamma(g)$ reduces to a finite Blaschke product $B_{g}$, then the closure of $\Gamma(g) H^{p}$ is $B_{g} H^{p}$. Since a function $f \in H^{p}$ is divisible by $B_{g}$ if and only if it vanishes on the zeroes of $B_{g}$ to an order greater or equal than that of $B_{g}, B_{g} H^{p}$ is then a subspace of finite codimension.

So Theorem 6 has as an immediate consequence the converse statements to those in Corollary 5 , and together they make up Theorem B from the introduction.

Corollary 7. (1) $\mathcal{A}$ is dense in $H^{p}$ if and only if $\Gamma(g)$ is an outer function.

(2) $\overline{\mathcal{A}}$ has finite codimension in $H^{p}$ if and only if the inner part of $\Gamma(g)$ is a finite Blaschke product (and in particular there is no singular inner factor).

An application of Theorem 6 to the polynomial case gives the following extension of the results proved in [3].

Let $p(z)$ and $q(z)$ be two polynomials. An argument similar to the one above alows to define $\mathcal{D}_{p}(q)$ and $\Gamma_{p}(q)$ by

$$
\begin{aligned}
& \mathcal{D}_{p}(q)(z):=\prod_{j=1}^{n-1}\left(q(z)-q\left(\varphi_{j}(z)\right)\right. \\
& \Gamma_{p}(q)(z):=\mathcal{D}_{p}(q)(z) / \mathcal{D}_{p}(z)
\end{aligned}
$$

where again $\left\{z=\varphi_{0}(z), \varphi_{1}(z), \ldots, \varphi_{n-1}(z)\right\}$ are $n$ roots of the polynomial equation (in t) $p(t)=p(z)$, and $n=\operatorname{deg} p$. The same argument shows that $\mathcal{D}_{p}(q)$ and $\Gamma_{p}(q)$ are polynomials.

The following result has Theorem $\mathrm{C}$ as a special case.

Theorem 8. Let $p_{1}, \ldots, p_{m} \in \mathbb{C}[z]$. Suppose that for some $1 \leq j \leq n$, at least one of $\Gamma_{p_{j}}\left(p_{k}\right), \quad k=1,2, \ldots, m, k \neq j$, is not identically zero. Then $\overline{\mathcal{A}}=\overline{\mathbb{C}}\left[p_{1}, \ldots, p_{m}\right]$ has finite codimension in $H^{2}$, and every closed $\mathcal{A}$-submodule of $H^{2}$ has at most $\min _{k \neq j}\left\{\mathcal{N}\left(\Gamma_{p_{j}}\left(p_{k}\right)\right)\right\}$ generators, where for a polynomial $R, \mathcal{N}(R)$ is the number of zeros of $R$ in the unit disk.

Proof. Let $M$ be a positive number large enough so that the interior $\Omega$ of some component of the lemniscate $\left\{z:\left|p_{j}(z)\right|=M\right\}$ contains the unit disk, and $p^{\prime}(z) \neq 0$ whenever $z \in \partial \Omega$. Denote by $\psi$ the Riemann mapping from $\Omega$ onto the unit disk , which maps $p_{j}(0)$ to the origin. Then $\psi$ is analytic in $\bar{\Omega}$ and the Hardy space in $\Omega$, $H^{2}(\Omega)$ (we equip $\partial \Omega$ with harmonic measure), is isometric to $H^{2}$ in the unit disk: an isometry is being given by

$$
F: f \longmapsto f \circ \psi
$$


ALGEBRAS GENERATED BY TWO BOUNDED HOLOMORPHIC FUNCTIONS 11

Note that $F\left(p_{j}\right)$ is a finite Blaschke product and $F\left(p_{l}\right), l=1, \ldots, m$, are analytic in a neighborhood of the unit disk. This implies that $\Gamma_{F\left(p_{j}\right)}\left(F\left(p_{k}\right)\right)=F\left(\Gamma_{p_{j}}\left(p_{k}\right)\right)$ have trivial singular parts in their inner-outer factorization for all $k=1, \ldots, m$ and their inner parts are finite Blaschke products. By Theorem $6, F(\overline{\mathcal{A}})$ contains $z$-invariant subspaces generated by these Blaschke products. In particular this implies that for every fixed $k, \mathcal{A}$ contains all polynomials vanishing with greater or equal multiplicity at all zeros of $\Gamma_{p_{j}}\left(p_{k}\right)$ in $\Omega$. Since the $H^{2}(\Omega)$ convergence implies $H^{2}$-convergence in the unit disk, Lemma 3 of [3] implies that the closure of $\mathcal{A}$ in $H^{2}$ contains the $z$-invariant subspace determined by the zeros of $\Gamma_{p_{j}}\left(p_{k}\right)$ which lie in the interior of the unit disk, and, therefore, has finite codimension. Let $B_{k}$ be the inner part of $\Gamma_{p_{j}}\left(p_{k}\right)$. Thus, since every $\mathcal{A}$-submodule $S$ of $H^{2}$ is a $\mathbb{C}\left[B_{k}\right]$-submodule, the number of generators of $S$ as $\mathcal{A}$-module does not exceed the number of generators of $S$ as $\mathbb{C}\left[B_{k}\right]$-module. By the Wold decomposition theorem this last number does not exceed the order of $B_{k}$ (see [3] for the details).

Corollary 9. If $\Gamma_{p_{j}}\left(p_{k}\right), k=1, \ldots, m, k \neq j$ have no common zeros in the unit disk, then $\mathcal{A}=\mathbb{C}\left[p_{1}, \ldots, p_{m}\right]$ is dense in $H^{2}$.

Proof. Let $a_{1}, \ldots, a_{N}$ be common zeros in $\mathbb{C}$ of the polynomials $\Gamma_{p_{j}}\left(p_{k}\right)$. There are polynomials $q_{k}, k=1, \ldots, m, k \neq j$ such that

$$
\sum_{k} \Gamma_{p_{j}}\left(p_{k}\right)(z) q_{k}(z)=R(z)=\prod_{l=1}^{N}\left(z-a_{l}\right) .
$$

By Theorem $6 \overline{R(z) H^{2}}$ is in $\overline{\mathcal{A}}$. Since $\left|a_{l}\right| \geq 1, R(z)$ is outer, and $\overline{R(z) H^{2}}=H^{2}$.

Note that in general the estimate given by Theorem 8 is sharp. As an example consider the algebra $\mathcal{A}=\mathbb{C}\left[p_{1}, p_{2}\right]$ where $p_{1}(z)=z(z-a), p_{2}(z)=z^{2}(z-a)$ for some $|a|<1$. It is easily seen that $\Gamma_{p_{1}}\left(p_{2}\right)(z)=p_{1}(z)$. Thus, by Theorem 8 every closed $\mathcal{A}$-submodule of $H^{2}$ has at most two generators. Let $M$ be the subspace of $H^{2}$ consisting of all functions vanishing at some point $b$ in the unit disk, different from $a$ and 0 . Clearly $M$ is a closed $\mathcal{A}$-submodule which is not singly generated. Indeed, for every function $g \in M$ such that $g(0) \neq 0$ and $g(a) \neq 0$, there exists a function $h \in M$ such that $g(0) h(a) \neq h(0) g(a)$, and, therefore, $g$ does not generate $M$ as $\mathcal{A}$-module. If $g(0)=0$ or $g(a)=0$, then since for any $f \in \mathcal{A}, f(0)=f(a)=0$, the $\mathcal{A}$-module generated by $g$ cannot contain $z-b$, for instance.

\section{CASE $\Gamma(g) \equiv 0$.}

At the other end of the spectrum from the density cases, there are cases when $\overline{\mathcal{A}}$ cannot contain any non-trivial invariant subspace - the simplest case would be when $B$ and $g$ are both even functions, for instance: then any function in $\overline{\mathcal{A}}$ is also even, and multiplying by $z$ takes us out of that subset. In that case, naturally, $\Gamma(g)$ vanishes identically, so Theorem 6 becomes vacuous. One would then like to know when $\Gamma(g) \equiv 0$.

A typical example of a situation when $\Gamma(g) \equiv 0$ is when there exists a function $u$ such that $B=B_{1} \circ u$ and $g=g_{1} \circ u$, and such that there exists a non-empty open set $\Omega \subset \mathbb{D}$ such that for any $z \in \Omega$, there exists $z^{\prime} \neq z, z^{\prime} \in \mathbb{D}$, such that $u\left(z^{\prime}\right)=u(z)$. 
This implies that $B\left(z^{\prime}\right)=B(z)$ and $g\left(z^{\prime}\right)=g(z)$, therefore $\Gamma(g)$ vanishes on $\Omega$, and thus on $\mathbb{D}$.

Theorem D states that this is how the identical vanishing of $\Gamma(g)$ always arises; it follows immediately from the following theorem.

Let us introduce a notation for the (finite) set of $z$ such that $\left\{\varphi_{j}(z), 0 \leq j \leq n-1\right\}$ is made up of fewer than $n$ points:

$$
\mathcal{S}_{0}:=\left\{z: B^{\prime}\left(\varphi_{j}(z)\right)=0 \text { for some } 0 \leq j \leq n-1\right\} .
$$

Theorem 10. The function $\Gamma(g)$ vanishes identically on the unit disk if and only if there exists an open set $\Omega \subset \mathbb{D} \backslash \mathcal{S}_{0}$ such that $\mathbb{D} \backslash \Omega$ is discrete, and an integer $m \geq 2$ such that for any $z \in \Omega$, the set $B^{-1}(B(z))$ is partitioned into subsets $A_{p}$ of cardinality $m$ such that $g$ is constant on each $A_{q}$.

Before passing to the proof of this theorem, notice that this means in particular that $m$ must divide $n$, therefore we obtain the following

Corollary 11. If $n$ is prime, $\Gamma(g) \equiv 0$ implies that $g$ must in fact be a function of $B$, and $\overline{\mathcal{A}}=\overline{\mathbb{C}[B]}$.

Another immediate corollary to Theorem 10 is the following result.

Corollary 12. The function $\Gamma(g)$ vanishes identically in the unit disk if and only if the only z-invariant subspace of $H^{p}$ contained in $\overline{\mathcal{A}}$ is the trivial subspace $\{0\}$.

Proof. If $\Gamma(g) \not \equiv 0$, a non-trivial $z$-invariant subspace contained in $\overline{\mathcal{A}}$ is given by Theorem 6 .

If $\Gamma(g) \equiv 0$ and $M$ is a nontrivial $z$-invariant subspace contained in $\overline{\mathcal{A}}$, choose $f \in M \backslash\{0\}$. Choose a point $a \in \Omega \backslash\{0\}$ such that $f(a) \neq 0$; then for any $b \neq a$ such that $B(b)=B(a)$ and $g(b)=g(a)$ (the above Theorem guarantees the existence of such points), we must have $f(b)=f(a)$, but $b f(b) \neq a f(a)$, which shows that $z f \notin \overline{\mathcal{A}}$, thus $z f \notin M$ and $M$ is not $z$-invariant.

Proof of Theorem 10. Define an integer-valued function in the disk by

$$
C(z):=\#\left\{w \in B^{-1}(B(z)): g(w)=g(z)\right\} .
$$

The hypothesis says that $C(z) \geq 2$ for any $z \in \Omega_{0}:=\mathbb{D} \backslash \mathcal{S}_{0}$.

We claim that $C$ is upper-semicontinuous on $\Omega_{0}$.

Indeed, let $z_{0} \in \Omega_{0}$, and let $\left\{z_{\nu}\right\}$ a sequence tending to $z_{0}$ such that $\lim _{\nu \rightarrow \infty} C\left(z_{\nu}\right)=$ $\lim \sup _{z \rightarrow z_{0}} C(z)$. We may assume that $\left\{z_{\nu}\right\}$ is contained in a neighborhood of $z_{0}$ on which $\varphi_{j}, 1 \leq j \leq n-1$ are all well-defined and distinct. For each $\nu \in \mathbb{Z}_{+}$, there exists a subset $A_{\nu} \subset\{0, \ldots n-1\}$ such that $g \circ \varphi_{j}\left(z_{\nu}\right)=g\left(z_{\nu}\right)$ if and only if $j \in A_{\nu}$, and $\# A_{\nu}=C\left(z_{\nu}\right)$. For $\nu$ large enough, this is constant and equal to $\lim \sup _{z \rightarrow z_{0}} C(z)$. Since there is only a finite number of subsets of $\{0, \ldots n-1\}$, we may also assume, by passing to a subsequence again denoted by $\left\{z_{\nu}\right\}$, that $A_{\nu}=A$ is a constant subset. Therefore for any $j \in A$,

$$
g \circ \varphi_{j}\left(z_{0}\right)=\lim _{\nu \rightarrow \infty} g \circ \varphi_{j}\left(z_{\nu}\right)=\lim _{\nu \rightarrow \infty} g\left(z_{\nu}\right)=g\left(z_{0}\right) .
$$

So $C\left(z_{0}\right) \geq \lim \sup _{z \rightarrow z_{0}} C(z)$, as claimed. 
ALGEBRAS GENERATED BY TWO BOUNDED HOLOMORPHIC FUNCTIONS 13

Let $m:=\min _{z \in \Omega_{0}} C(z) \geq 2$. This makes sense since $C$ is integer-valued, and since it is u.s.c. the set

$$
\Omega_{1}:=\left\{z \in \Omega_{0}: C(z)=m\right\}
$$

is open.

We claim that $E:=\Omega_{0} \backslash \Omega_{1}$ (and therefore $\mathbb{D} \backslash \Omega_{1}$ ) only has isolated points.

Indeed, let $E^{\prime}$ be the set of non-isolated points within $E$. Since $E$ is closed in $\Omega_{0}$, so is $E^{\prime}$. On the other hand, suppose $z_{0} \in E^{\prime}$. Restrict attention to a neighborhood of $z_{0}$ where a continuous choice of $\varphi_{j}$ can be made. Then, reasoning as in the proof of the previous claim, we can find a sequence $\left\{z_{\nu}\right\} \subset E$ tending to $z_{0}$ and a set $A \subset\{0, \ldots n-1\}$ such that $g \circ \varphi_{j}\left(z_{\nu}\right)=g\left(z_{\nu}\right)$ if and only if $j \in A$. Then, for any $j \in A \backslash\{0\}$, the analytic function $g-g \circ \varphi_{j}$ vanishes on a subset which has a limit point, and therefore is identically 0 in a small disk $z_{0}$. This proves that any point in this disk is in $E$, and therefore in $E^{\prime}$. So $E^{\prime}$ is also an open set. Since $\Omega_{0}$ is connected, if $E^{\prime} \neq \emptyset$, then $E^{\prime}=\Omega_{0}$, which is absurd. Therefore $E^{\prime}=\emptyset$, q.e.d.

Now let

$$
\Omega_{2}:=\left\{z \in \Omega_{1}: \varphi_{j}(z) \in \Omega_{1}, 1 \leq j \leq n-1\right\} .
$$

Then $\Omega_{0} \backslash \Omega_{2}$ (and therefore $\mathbb{D} \backslash \Omega_{2}$ ) only has isolated points.

Indeed, $z \in \Omega_{0} \backslash \Omega_{2}$, if and only if there is some $j \in\{0, \ldots, n-1\}$ such that $w=\varphi_{j}(z), w \in \Omega_{0} \backslash \Omega_{1}$. Take a point $z_{0} \in \Omega_{0}$, and a compact neighborhood of $U$ of $z_{0}$; then $B^{-1}(B(U))$ is a compact set (since a finite Blasche product is a proper map from the disk to itself), therefore can only contain a finite number of points from $\Omega_{0} \backslash \Omega_{1}$ by the above claim, so there can only be a finite number of points in $\Omega_{0} \backslash \Omega_{2}$ in $U$, q.e.d.

For any point $z \in \Omega_{2}$, the fiber $B^{-1}(B(z))$ is made up of exactly $n$ points, and each point in this fiber is part of a group of $m$ points for which the values of $g$ coincide. So the theorem holds with $\Omega=\Omega_{2}$.

\section{6. z-INVARIANT SUBSPACES IN THE ALGEBRA'S CLOSURE}

In general one may ask, first, how large can be the $z$-invariant subspaces contained in the closure of $\mathcal{A}=\mathbb{C}[B, g]$ ? and, second, can $\mathbb{C}[B, g]$ be included in some closed space that is easy to describe in terms of the well-understood $\mathbb{C}[B]$ and the function $\Gamma(g)$ ? In the introduction both questions were answered in the case of Blaschke products of degree two. In this section we consider the case of Blaschke products of higher degree.

As to the second question, all we can say for now is that $\Gamma(g)$ can be expressed as a polynomial of degree at most $n-1$ in $g$ with coefficients in $\overline{\mathbb{C}[B]}$ (this follows from the proof of Theorem 6 , see below). Therefore all elements of $\mathbb{C}[B, g]$ are algebraic of degree at most $n-1$ over the ring $\overline{\mathbb{C}[B]}[\Gamma(g)] \subset \overline{\mathbb{C}[B]}+\overline{\Gamma(g) H^{p}}$.

The first question is not easy to answer in full generality. The following examples, reflecting respectively cases where the inner factor of $\Gamma(g)$ has either zeros inside the disk and singular factors at the boundary, suggest that $\Gamma(g) H^{p}$ is optimal in certain cases.

Example 1. Suppose $B(z)=z^{n}$ and $g(z)=z^{m}$. 
Then if the greatest common divisor of $n$ and $m$ is $\delta \geq 2$, both $B$ and $g$ are expressed in terms of the function $u(z)=z^{\delta}, \Gamma(g)(z) \equiv 0$ and $\overline{\mathcal{A}}$ contains no nontrivial invariant subspace.

So assume that $n$ and $m$ are relatively prime. Then $\Gamma(g)(z)=c z^{(n-1)(m-1)}$, where $c=c(n, m)$ is a nonzero complex constant.

Here $\mathbb{C}[B, g]$ is the span of the monomials $\left\{z^{k}, k \in A\right\}$, where $A:=\{a n+b m, a, b \in$ $\left.\mathbf{Z}_{+}\right\}$, and its closure is made up of the functions $f \in H^{p}$ such that $f^{(k)}(0)=0$ for $k \notin A$.

It is easy to see that the largest invariant subspace that can be contained in the closure of this algebra will be of the form $z^{N} H^{2}$, where $N$ is the smallest integer such that any $\{N, N+1, \ldots\} \subset A$. It is a classical result in arithmetic that $N=$ $(n-1)(m-1)(\mathrm{cf}[8$, Theorem 1.17, p. 39], see also [2]).

Note also that in this case the full algebra $\mathbb{C}[B, g]$ is obtained by adjoining the $(n-1)$-th root of $\Gamma(g)$ to $\mathbb{C}[B]$ (so in this case the comments made above about the second question give the complete answer). The codimension of the closure of $\mathbb{C}[B, g]$ is $(1 / 2)(n-1)(m-1)$ (cf [8, exercise 7, p. 41], or [2]).

Example 2. Let $B(z)=z^{n}$ and $g(z)=z \chi\left(z^{n}\right)$ (as above $\chi$ is the singular inner function determined by the unit point mass at 1). Then $\Gamma(g)(z)=\chi\left(z^{n}\right)^{n-1}=S_{g}(z)$. Note that for any $\alpha<1 /(n-1), \Gamma(g)^{\alpha} H^{p} \not \subset \overline{\mathcal{A}}$.

Indeed, let $f(z):=z \Gamma(g)(z)^{\alpha}=z \chi\left(z^{n}\right)^{(n-1) \alpha}$, then $\Gamma(f)(z)=\chi\left(z^{n}\right)^{(n-1)^{2} \alpha}$, which is not divisible by $S_{g}$ since $(n-1)^{2} \alpha<n-1$. Thus, by Proposition $4, f \notin \overline{\mathcal{A}}$.

There is a partial necessary condition about the vanishing of any inner function $J$ such that $J H^{p} \subset \overline{\mathcal{A}}$.

Proposition 13. Let $\overline{\mathcal{A}}=\overline{\mathbb{C}[B, g]}, B$ a Blaschke product of degree $n$. Suppose that $J H^{p} \subset \overline{\mathcal{A}}$, where $J$ is inner. Then, for any $a \in \mathbb{D}$ such that $\Gamma(g)(a)=0$, we have $J(a)=0$.

Proof. Let $a$ be a zero of $B_{g}$ (recall that $B_{g}$ is the Blaschke part of the canonical factorization of $\Gamma(g))$.

Case 1. Suppose that $a \neq \varphi_{j}(a)$, for $1 \leq j \leq n-1$.

Then set $f(z):=\prod_{1}^{n-1}\left(z-\varphi_{j}(a)\right)$. By our hypothesis, $f J \in \overline{\mathcal{A}}$, so by Proposition ๑, $\Gamma(f J)$ is divisible by $B_{g}$.

We have

$$
\Gamma(f J)(z)=\prod_{j=1}^{n-1} \frac{f(z) J(z)-f \circ \varphi_{j}(z) J \circ \varphi_{j}(z)}{z-\varphi_{j}(z)},
$$

and $f \circ \varphi_{j}(a)=0$ for $1 \leq j \leq n-1$. So

$$
\Gamma(f J)(a)=\frac{(f(a) J(a))^{n-1}}{\prod_{j=1}^{n-1}\left(a-\varphi_{j}(a)\right)}=0,
$$

which implies $J(a)=0$.

Case 2. Suppose that $a=\varphi_{j}(a)$ for some $j$.

Now $B_{g}(a)=0$ is due to the fact that $B^{\prime}(a)=g^{\prime}(a)=0$. Then any $f \in \overline{\mathcal{A}}$ must satisfy $f^{\prime}(a)=0$, but if we had $J(a) \neq 0$, then for $f(z):=z J(z)$ we would have $f^{\prime}(a) \neq 0$, so $J H^{p} \not \subset \overline{\mathcal{A}}$. 


\section{ALGEBRAS GENERATED BY TWO BOUNDED HOLOMORPHIC FUNCTIONS 15}

\section{Applichtions to the Disk Algebra}

Let us consider the special case where the function $g$ is a member of the disk algebra, that is to say $g \in A(\overline{\mathbb{D}}):=\mathcal{O}(\mathbb{D}) \cap \mathcal{C}(\overline{\mathbb{D}})$, the algebra of holomorphic functions continuous up to the boundary of the disk, endowed with the uniform norm on $\overline{\mathbb{D}}$. One is now interested in $\overline{\mathcal{A}}$, where the closure is taken in $A(\overline{\mathbb{D}})$.

The closure of $\mathbb{C}[f, g]$ in $A(\overline{\mathbb{D}})$ has been studied by several authors, see e.g. [11], [1]. We can apply the methods of the previous sections in the case when $g$ is continuous up to the unit circle. Notice in particular that then $\Gamma(g) \in A(\overline{\mathbb{D}})$, and whenever $\Gamma(g)$ is zero-free, $\Gamma(g) A(\overline{\mathbb{D}})=A(\overline{\mathbb{D}})$.

Theorem 14. $\overline{\mathcal{A}}$ contains $\Gamma(g) A(\overline{\mathbb{D}})$.

Corollary 15. (1) $\mathcal{A}$ is dense in $A(\overline{\mathbb{D}})$ if and only if $\Gamma(g)$ has no zeros in the closed unit disk (in particular $\Gamma(g)$ is then outer).

(2) $\overline{\mathcal{A}}$ has finite codimension in $A(\overline{\mathbb{D}})$ if and only if $\Gamma(g)$ has no singular inner factor, and only a finite number of zeros in the closed unit disk.

The proofs of both results rely on the proof of Theorem [6 so we will postpone them until the end of section 8 .

\section{Comparison with previous results}

The earliest results which we know in this direction have been obtained by John Wermer in [11], where the uniform closure of an algebra of the form $\mathbb{C}[f, g]$ was considered, with $f, g$ analytic in a neighborhood of the closed disk (actually, Wermer's results hold in the more general context of a domain bounded by a simple curve on a Riemann surface) and $f^{\prime}(\zeta) \neq 0$ for any $\zeta \in \partial \mathbb{D}$.

Then Theorem 1.1 in 11 states that $\overline{\mathbb{C}[f, g]}=A(\overline{\mathbb{D}})$ if and only if the algebra separates points of the closed disk and the derivatives of the generators never vanish simultaneously.

In our case (where $f=B$, a finite Blaschke product), by Lemma 2, this is equivalent to $\Gamma(g)$ having no zeros on the closed disk (a condition which automatically rules out singular inner factors). So a more special hypothesis on the function $f$ helps us relax considerably the hypotheses on $g$.

It should be noted that consideration of the function $g_{r}(z):=g(r z)$, for $r<1$, which is analytic in a neighborhood of the closed disk, does not help in this instance, because the convergence of $g_{r}$ to $g$ is not enough to ensure the corresponding "convergence" of the closed algebras they generate.

For instance, consider the following example:

$$
B(z)=z^{2}, \quad g(z)=z\left(1-z^{2}\right) \chi\left(z^{2}\right),
$$

where $\chi$ stands for the same singular inner function as in the examples of the introduction. Then $\Gamma(g)(z)=\left(1-z^{2}\right) \chi\left(z^{2}\right)$, so it has a singular inner factor and $\overline{\mathbb{C}[B, g]}$ is of infinite codimension. On the other hand, $\Gamma\left(g_{r}\right)(z)=r\left(1-r^{2} z^{2}\right) \chi\left(r^{2} z^{2}\right)$ has no zeros on the closed disk for any $r<1$, so then $\overline{\mathbb{C}\left[B, g_{r}\right]}=A(\overline{\mathbb{D}})$.

In [1], more general algebras $\mathcal{A}$ are considered. If $\mathcal{A}$ separates points of the closed disk, contains a dense subalgebra of functions which are $\mathcal{C}^{1}$ up to the boundary, and for any $z \in \overline{\mathbb{D}}$ there is $f_{z} \in \mathcal{A}$ such that $f_{z}^{\prime}(z) \neq 0$, then $\mathcal{A}$ is dense in $A(\overline{\mathbb{D}})$. Actually, 
this immediate consequence of Blumenthal's work is quoted thus in [12], a paper which summarizes and slightly generalizes an unpublished dissertation [4]. As far as we know, those were the first works to point out the role as obstructions to density of singular inner factors of functions analogous to our $\Gamma(g)$.

For algebras of the special form $\overline{\mathbb{C}[B, g]}$, Blumenthal's result extends Wermer's theorem to the case where $g \in \mathcal{C}^{1}(\overline{\mathbb{D}})$. In this case, our methods can cover further examples of functions $g$ which yield a dense subalgebra of $A(\overline{\mathbb{D}})$. For instance, consider

$$
B(z)=z^{2}, \quad g(z)=(1-z) \chi(z) \in A(\overline{\mathbb{D}}) \backslash \mathcal{C}^{1}(\overline{\mathbb{D}}) .
$$

Then

$$
\Gamma(g)(z)=\frac{1}{2 z}((1-z) \chi(z)-(1+z) \chi(-z)) .
$$

One checks easily that $\Gamma(g)(0) \neq 0$.

In fact, $\Gamma(g)$ never vanishes. For $z \neq 0$, setting $\sigma:=(1+z) /(1-z)$, we see that $\Gamma(g)(z)=0$ if and only if

$$
\exp \left(\frac{1}{\sigma}-\sigma\right)=\sigma
$$

and since $\operatorname{Re}\left(\frac{1}{\sigma}-\sigma\right)>0$ if and only if $|\sigma|<1$, any solutions would have to be of the form $\sigma=e^{i \theta}$, which leads to $e^{-2 i \sin \theta}=e^{i \theta}$, an equation whose only solution in the relevant interval $\left(-\frac{\pi}{2}, \frac{\pi}{2}\right)$ is $\theta=0$, which corresponds to $z=0$, excluded.

Furthermore, $\Gamma(g)$ is analytic in a neighborhood of $\overline{\mathbb{D}} \backslash\{+1,-1\}$, so any singular inner factor would have its singular mass carried by $\{+1,-1\}$. However, one verifies immediately that $\Gamma(g)$ has nonzero limits at each of those points, so that it cannot admit any singular factor. Therefore, by Corollary $15(\mathrm{i}), \overline{\mathbb{C}[B, g]}=A(\overline{\mathbb{D}})$.

The papers of Wermer and Blumenthal cited above also include results of the same type as Theorem 14, whose conclusion is that the closure of a given algebra contains a certain ideal of finite codimension.

Theorem 1.2 in 111 states that, under the same regularity hypotheses on $f, g$, if $\mathbb{C}[f, g]$ separates points on $\partial \mathbb{D}$, then $\overline{\mathbb{C}[f, g]}$ contains a subspace of the form $P A(\overline{\mathbb{D}})$, where $P$ is a polynomial. It should be noted that the hypothesis about pointseparation is easily weakened here to the hypothesis that the set of points which are not separated be discrete; then a small change in the radius of the boundary circle (legitimate since our functions are analytic in a neighborhood of the closed disk) will produce a situation where the points of the circle are separated.

In the case of $\mathbb{C}[B, g]$, if $g$ is analytic in a neighborhood of the closed disk, then so is $\Gamma(g)$, so it can't have singular inner factors, nor an infinite number of zeros on the closed disk unless it is identically zero. That last occurrence rules out any non-trivial closed ideal being contained in $\mathbb{C}[B, g]$, by the proof of Corollary 12 .

Blumenthal's Theorem 1 in [1] extends Wermer's theorem to cases where $g \in \mathcal{C}^{1}(\overline{\mathbb{D}})$ only; in view of Theorem 14 this assumption is not needed when considering $\mathbb{C}[B, g]$. (Furthermore, Blumenthal's theorem includes a hypothesis of point-separation which is stronger than expected for the conclusion obtained).

An outstanding problem is the determination of $\overline{\mathbb{C}[f, g]}$ when $f$ and $g \in A(\overline{\mathbb{D}})$, even if $f$ is assumed to be analytic in a neighborhood of $\overline{\mathbb{D}}$ (but no extra hypotheses are imposed on $g$ ). 


\section{Proof of the Main Theorem}

Proof of Theorem 6. Suppose $f \in H^{p}$, let $f_{r}(z)=f(r z)$ for $r<1$. If a sequence $f_{\nu}$ can be found in $\mathcal{A}$ which approximates $f_{r}$ pointwise boundedly, then $f_{r}$ belongs to the weak closure of $\mathcal{A}$ in $H^{p}$. Since the weak closure of $\mathcal{A}$ coincides with the strong closure, $f_{r}$ is in the $H^{p}$-closure of $\mathcal{A}$. The proof of Theorem 6 therefore reduces to the following Proposition.

Proposition 16. For any $h \in \Gamma(g) H^{\infty}$, there is a sequence $\left\{h_{\nu}\right\} \subset \mathcal{A}$ converging to $h$ pointwise boundedly.

Proof. First we need to introduce notations for two kinds of singularities (in a certain sense) that we will encounter. Recall from (2) that $\mathcal{S}_{0}$ is the (finite) set of $z$ such that $\left\{\varphi_{j}(z), 0 \leq j \leq n-1\right\}$ is made up of fewer than $n$ points.

Larger still is the set $\mathcal{S}_{1}$ of $z$ such that $\left\{g\left(\varphi_{j}(z)\right), 0 \leq j \leq n-1\right\}$ is made up of fewer than $n$ points. The previous considerations show that $\mathcal{S}_{1} \backslash \mathcal{S}_{0}$ is contained in the zero set of $\Gamma(g)$, so that if it is not discrete, $\Gamma(g) \equiv 0$, and our theorem is vacuously true.

Let $h$ be any holomorphic function in the disc (later we will choose $h=f \Gamma(g)$ ).

Restrict attention for a moment to points $z \in \mathbb{D} \backslash \mathcal{S}_{1}$. Then there is a unique polynomial $\mathcal{L}$ of degree not exceeding $n-1$ such that

$$
\mathcal{L}\left(g \circ \varphi_{j}(z)\right)=h\left(\varphi_{j}(z)\right), 0 \leq j \leq n-1 .
$$

The polynomial $\mathcal{L}$ can be written $\mathcal{L}(X)=\sum_{k=0}^{n-1} \alpha_{j}(B(z)) X^{k}$, since its coefficients depend only on the set $B^{-1}(B(z))$, and, being symmetric in the elements of that set and given by fractions (Lagrange's interpolation formula), are holomorphic in $\mathbb{D} \backslash \mathcal{S}_{1}$. So we have, at any such point $z$,

$$
h(z)=\sum_{j=0}^{n-1} \alpha_{j}(B(z)) g(z)^{j} .
$$

Now it suffices to show that when $h=f \Gamma(g)$, the $\alpha_{j}$ turn out to be bounded analytic functions in the unit disk. We write $\mathcal{L}=\mathcal{L}_{z}$ to emphasize the dependency on $z$.

In a punctured neighborhood of any point of $\mathcal{S}_{1}, \mathcal{L}$ is given by the Lagrange formula,

$$
\mathcal{L}_{z}(X)=\sum_{j=0}^{n-1} f\left(\varphi_{j}(z)\right) \Gamma(g)\left(\varphi_{j}(z)\right) \prod_{l: l \neq j} \frac{X-g\left(\varphi_{l}(z)\right)}{g\left(\varphi_{j}(z)\right)-g\left(\varphi_{l}(z)\right)} .
$$

On the other hand,

$$
\Gamma(g)\left(\varphi_{j}(z)\right)=\prod_{i=1}^{n-1} \frac{g\left(\varphi_{j}(z)\right)-g\left(\varphi_{i} \circ \varphi_{j}(z)\right)}{\varphi_{j}(z)-\varphi_{i} \circ \varphi_{j}(z)} .
$$

When $i$ goes through the set $\{1, \ldots, n-1\}, \varphi_{i} \circ \varphi_{j}(z)$ goes through the set $\left\{\varphi_{l}(z), l \neq\right.$ $j\}$, so the above numerators cancel out with the denominators (3). So finally

$$
\mathcal{L}_{z}(X)=\sum_{j=0}^{n-1} f\left(\varphi_{j}(z)\right) \prod_{l: l \neq j} \frac{X-g\left(\varphi_{l}(z)\right)}{\varphi_{j}(z)-\varphi_{l}(z)}
$$


This formula makes sense whenever $z \notin \mathcal{S}_{0}$, and shows that the coefficients of $\mathcal{L}$ are holomorphically extendable to $\mathbb{D} \backslash \mathcal{S}_{0}$.

Consider now a neighborhood $V$ of the unit circle chosen so that its closure is disjoint from $\mathcal{S}_{0}$. For $z \in V,\left|\varphi_{j}(z)-\varphi_{k}(z)\right|$ is bounded below. So to prove that the $\alpha_{j}$ are bounded analytic functions, it will be enough to prove that the singularities for $z \in \mathcal{S}_{0}$ are removable.

This will be done by considering several cases in turn for the possible singularity $z_{0} \in \mathcal{S}_{0}$. First, we consider a type of function $g$ which allows us to describe explicitly what the interpolating polynomial $\mathcal{L}_{z}$ turns out to be when $z$ is a critical point of $B$.

Case 1. $g^{\prime}(z) \neq 0$ for any $z \in B^{-1}\left(B\left(z_{0}\right)\right)$, and $g$ is one-to-one on the set $B^{-1}\left(B\left(z_{0}\right)\right)$.

Note that this hypothesis is equivalent to the fact that $g$ is one-to-one in some neighborhood $U$ of $B^{-1}\left(B\left(z_{0}\right)\right)$.

Then $g^{-1}$ is holomorphic on the open set $g(U)$, and $\mathcal{L}$ is exactly the interpolation polynomial of degree less or equal than $n-1$ assuming the same values as $h \circ g^{-1}$ at the points of $g \circ B^{-1}(B(z))$, for $z \in U \backslash \mathcal{S}_{0}$. Choose a smaller neighborhood $U_{0}$ of $B^{-1}\left(B\left(z_{0}\right)\right), U_{0}$ relatively compact in $U$, such that the boundary of $g\left(U_{0}\right)$ is a finite disjoint union of circles. For $z$ belonging to a small enough neighborhood of $z_{0}$ such that $B^{-1}(B(z)) \subset U_{0}$, and for $x \in B\left(U_{0}\right)$,

$$
\mathcal{L}_{z}(x)=\frac{1}{2 \pi i} \int_{\partial U_{0}} \frac{h \circ g^{-1}(\zeta)}{\omega_{z}(\zeta)} \frac{\omega_{z}(\zeta)-\omega_{z}(x)}{\zeta-x} d \zeta
$$

where $\omega_{z}(X):=\prod_{0 \leq l \leq n-1}\left(X-g \circ \varphi_{l}(z)\right)$ [7, Vol. II, Section 11, p. 67 ff.]. This formula depends continuously on $z$ in the whole neighborhood $U_{0}$, and converges, when $z$ converges to $z_{0}$, to the solution of an Hermite interpolation problem given by

$$
\mathcal{L}^{(j)}\left(g \circ \varphi_{i}(z)\right)=h^{(j)}\left(\varphi_{i}(z)\right), \text { for } i \in A_{q}, 0 \leq j \leq \# A_{q}-1,1 \leq q \leq p,
$$

where $A_{1}, \ldots, A_{p}$ form a partition of $\{0, \ldots, n-1\}$ such that $\varphi_{j}(z)=\varphi_{j^{\prime}}(z)$ if and only if $j$ and $j^{\prime}$ belong to the same set $A_{q}$.

Case 2. For any point $z_{0}$ such that there exists a $j$ with $B^{\prime}\left(\varphi_{j}\left(z_{0}\right)\right)=0$, we have $B^{\prime \prime}\left(\varphi_{j}\left(z_{0}\right)\right) \neq 0$ for any such $j$.

This hypothesis says that the equation $B(z)=B\left(z_{0}\right)$ has solutions of multiplicity never exceeding 2. It does not depend on $g$. Notice that this case is generic: Blaschke products of degree greater than one always admit critical points inside the disk, and those for which multiplicities of the above solutions can be 3 or more form a small set, in a sense that will be made precise at the beginning of Case 3.

Then the various terms in the sum which represents $\mathcal{L}_{z}(X)$ either converge to a finite limit (when $j$ is such that $B^{\prime}\left(\varphi_{j}\left(z_{0}\right)\right) \neq 0$ ) or can be regrouped in pairs $(j, k)$ where $j \neq k, \varphi_{j}\left(z_{0}\right)=\varphi_{k}\left(z_{0}\right) \neq \varphi_{l}\left(z_{0}\right)$ for $l \notin\{j, k\}$. To simplify notations, let us assume that $j=0, k=1$. For $z$ in a small enough neighborhood of $z_{0}$, the sum of 


\section{ALGEBRAS GENERATED BY TWO BOUNDED HOLOMORPHIC FUNCTIONS 19}

the two terms can be written

$$
\begin{aligned}
& f(z) \frac{X-g \circ \varphi_{1}(z)}{z-\varphi_{1}(z)} \prod_{2 \leq l \leq n-1} \frac{X-g \circ \varphi_{l}(z)}{z-\varphi_{l}(z)} \\
& \quad+f \circ \varphi_{1}(z) \frac{X-g(z)}{\varphi_{1}(z)-z} \prod_{2 \leq l \leq n-1} \frac{X-g \circ \varphi_{l}(z)}{\varphi_{1}(z)-\varphi_{l}(z)} \\
& =\frac{\prod_{2 \leq l \leq n-1}\left(X-g \circ \varphi_{l}(z)\right)}{z-\varphi_{1}(z)}\left(\frac{f(z)\left(X-g \circ \varphi_{1}(z)\right)}{\prod_{2 \leq l \leq n-1}\left(z-\varphi_{l}(z)\right)}-\frac{f \circ \varphi_{1}(z)(X-g(z))}{\prod_{2 \leq l \leq n-1}\left(\varphi_{1}(z)-\varphi_{l}(z)\right)}\right) .
\end{aligned}
$$

Define a meromorphic function whose coefficients depend on $z$ by

$$
R(Y):=\frac{f(Y)}{\prod_{2 \leq l \leq n-1}\left(Y-\varphi_{l}(z)\right)} .
$$

The poles of $R$ depend on the parameter $z$ and stay away from $z_{0}$ for $z$ in a neighborhood of $z_{0}$, and we have

$$
R\left(Y_{1}\right)-R\left(Y_{2}\right):=\left(Y_{1}-Y_{2}\right) R_{1}\left(Y_{1}, Y_{2}\right),
$$

where $R_{1}$ is another meromorphic function (of two variables) with its pole set (which is a hypersurface of $\left.\mathbb{C}^{2}\right)$ avoiding a neighborhood of $\left(z_{0}, z_{0}\right)$. The expression from (5) is then equal to

$$
\begin{aligned}
& \frac{\prod_{2 \leq l \leq n-1}\left(X-g \circ \varphi_{l}(z)\right)}{z-\varphi_{1}(z)}\left[R(z)\left(X-g \circ \varphi_{1}(z)\right)-R\left(\varphi_{1}(z)\right)(X-g(z))\right] \\
= & \left(\prod_{2 \leq l \leq n-1}\left(X-g \circ \varphi_{l}(z)\right)\right)\left(R_{1}\left(z, \varphi_{1}(z)\right)\left(X-g \circ \varphi_{1}(z)\right)+\frac{g(z)-g \circ \varphi_{1}(z)}{z-\varphi_{1}(z)} R\left(\varphi_{1}(z)\right)\right) .
\end{aligned}
$$

Letting $z$ tend to $z_{0}, \varphi_{1}(z)$ tends to $z_{0}$ as well, and $\frac{g(z)-g \circ \varphi_{1}(z)}{z-\varphi_{1}(z)}$ tends to $g^{\prime}\left(z_{0}\right)$, so the coefficients of $\mathcal{L}_{z}$ admit finite limits, and the possible singularity of its coefficients at $z_{0}$ is actually removable.

Case 3. General case.

The Blaschke products of degree $n$ which do not satisfy the hypotheses of Case 2 form an algebraic subset, therefore of empty interior, since their derivative, which is a rational fraction of fixed degree, must admit at least one multiple root.

Given one such exceptional Blaschke product $B$, let $B^{\nu}$ be a sequence of Blaschke products satisfying the hypotheses of Case 2 and converging to $B$ uniformly in a neighborhood the closed unit disk. By continuous dependency of the roots of a polynomial equation upon the coefficients (Hurwitz Theorem), we see that the corresponding functions $\varphi_{j}^{\nu}$ converge to $\varphi_{j}$ uniformly in a some neighborhood of the unit circle (where they are all well-defined), and that any symmetric polynomial in the $\varphi_{j}^{\nu}$ 's converges uniformly in the closed disk to the same polynomial in the $\varphi_{j}$ 's. In particular, if we set $\Gamma^{\nu}(g)=\Gamma_{B^{\nu}}(g)$, then $\Gamma^{\nu}(g)$ converges to $\Gamma(g)$ pointwise boundedly on the disk. 
Given a function $f \in H^{\infty}(\mathbb{D})$, write

$$
\mathcal{L}_{z}^{\nu}(X)=\sum_{j=0}^{n-1} f\left(\varphi_{j}^{\nu}(z)\right) \Gamma^{\nu}(g)\left(\varphi_{j}^{\nu}(z)\right) \prod_{l: l \neq j} \frac{X-g\left(\varphi_{l}^{\nu}(z)\right)}{g\left(\varphi_{j}^{\nu}(z)\right)-g\left(\varphi_{l}^{\nu}(z)\right)}=: \sum_{k=0}^{n-1} A_{k}^{\nu}(z) X^{k}
$$

From (4) and the proof in Case 2, we see that the $A_{k}^{\nu}$ are bounded holomorphic functions and $A_{k}^{\nu}(z)=\alpha_{k}^{\nu}\left(B^{\nu}(z)\right)$.

Fix some degree $k$ between 0 and $n-1$. On any circle centered at the origin of radius $r$ close enough to $1, A_{k}^{\nu}$ converges uniformly, because $f$ is bounded on the circle, all the $\varphi_{j}^{\nu}$ are well defined and converge uniformly, and all the denominators in the expression are bounded away from 0 . If $f$ is furthermore bounded, the limit is a bounded holomorphic function which we denote by $A_{k}$.

We need to see that for any $z_{1}, z_{2}$ such that $B\left(z_{1}\right)=B\left(z_{2}\right)$, then $A_{k}\left(z_{1}\right)=A_{k}\left(z_{2}\right)$. In that case, there is a $j$ such that $\varphi_{j}\left(z_{1}\right)=z_{2}$, and by the convergence of the $\varphi_{j}^{\nu}$, we can select a subsequence (which we will denote the same way as the original sequence) and an index $j_{0}$ such that $z_{2}-\varphi_{j_{0}}^{\nu}\left(z_{1}\right) \rightarrow 0$ as $\nu \rightarrow \infty$. Then

$$
A_{k}\left(z_{1}\right)-A_{k}\left(z_{2}\right)=\lim _{\nu \rightarrow \infty}\left(A_{k}^{\nu}\left(z_{1}\right)-A_{k}^{\nu}\left(z_{2}\right)\right)=\lim _{\nu \rightarrow \infty}\left(A_{k}^{\nu}\left(\varphi_{j_{0}}^{\nu}\left(z_{1}\right)\right)-A_{k}^{\nu}\left(z_{2}\right)\right)=0,
$$

where the next to last equality is by the symmetry of the $A_{k}^{\nu}$, and the last by uniform convergence on compacta. So we can write $A_{k}(z)=\alpha_{k}(B(z))$, where $\alpha_{k} \in H^{\infty}(\mathbb{D})$. Now

$$
f(z) \Gamma(g)(z)=\lim _{\nu \rightarrow \infty} f(z) \Gamma^{\nu}(g)(z)=\lim _{\nu \rightarrow \infty} \sum_{k=0}^{n-1} A_{k}^{\nu}(z) g(z)^{k}=\sum_{k=0}^{n-1} \alpha_{k}(B(z)) g(z)^{k} .
$$

We now are able to give the proofs of the corresponding results for the disk algebra case.

Proof of Theorem 14. The proof proceeds exactly as the proof of Proposition 16, except that now we will need to assume $h=f \Gamma(g)$, with $f \in A(\overline{\mathbb{D}})$. This time we need to prove that the functions $\alpha_{j}$ are continuous up to the boundary (in addition to being holomorphic). For this it will be enough to show that the coeffients of $\mathcal{L}_{z}$ are themselves continuous up to the boundary. This follows from the formula (四), since $f$ and $g$ are continuous, and the $\varphi_{j}$ are all distinct near the unit circle.

Furthermore, the arguments of Case 3 now show that $\Gamma^{\nu}(g)$ converges to $\Gamma(g)$ uniformly on the closed disk (using the uniform continuity of $g$ on the closed disk). So the coefficients $A_{k}^{\nu}$ converge uniformly to functions in the disc algebra, and one proves in the usual way that the limits are functions of $B$ alone.

Proof of Corollary 15. (i) Any zero of $\Gamma(g)$ in the open disk provides a continuous, non-trivial linear form which vanishes on $\mathcal{A}$, by the proof of Corollary 5 . If $\Gamma(g)$ has a zero $\zeta$ on the boundary, then since $B^{\prime}$ never vanishes on the unit circle, there must exist $\zeta^{\prime} \in \partial \mathbb{D}, \zeta^{\prime} \neq \zeta$, such that $B(\zeta)=B\left(\zeta^{\prime}\right), g(\zeta)=g\left(\zeta^{\prime}\right)$. Then $f \mapsto f(\zeta)-f\left(\zeta^{\prime}\right)$ provides a non-trivial linear form, continuous on $A(\overline{\mathbb{D}})$, which vanishes on $\mathcal{A}$.

The converse is immediate by the remark made before the statement of Theorem 14. 


\section{ALGEBRAS GENERATED BY TWO BOUNDED HOLOMORPHIC FUNCTIONS 21}

(ii) As above, an infinite number of zeros of $\Gamma(\mathrm{g})$ would provide arbitrarily large (finite) sets of linearly independent continuous linear forms vanishing on $\mathcal{A}$, so the codimension could not be finite.

Suppose on the other hand that the factorization of $\Gamma(g)$ admit a singular inner factor $S$. It is easy to see that the map $f \mapsto \Gamma(f)$ is continuous on $A(\overline{\mathbb{D}})$, so that whenever $f \in \overline{\mathcal{A}}, \Gamma(f)$ is divisible by $\Gamma(g)$, therefore by $S$. Arguing as in the proof of Corollary 5 again, if $\overline{\mathcal{A}}$ was of finite codimension, it would contain a polynomial, which would lead to a contradiction.

Conversely, if $\# \Gamma(g)^{-1}\{0\}$ is finite, it will be enough to show that the codimension of the closure of $\Gamma(g) A(\overline{\mathbb{D}})$ is finite. Let $\mathcal{I}:=\overline{\Gamma(g) A(\overline{\mathbb{D}})}$. Then $\mathcal{I}$ is a closed ideal of $A(\overline{\mathbb{D}})$, and the main result of $[9]$ shows that $\mathcal{I}=I(E, M)$, the set of all functions in $A(\overline{\mathbb{D}})$ which vanish on the closed set $E \subset \partial \mathbb{D}$ and which are divisible by the inner function $M$. The hypothesis on $\Gamma(g)$ imply that $M$ is a finite Blaschke product (because singular inner factors are excluded), and that $E=\Gamma(g)^{-1}\{0\} \cap \partial \mathbb{D}$ is a finite subset of the circle. Therefore $\mathcal{I}$ reduces to those functions in $A(\overline{\mathbb{D}})$ which vanish on $E^{\prime}:=\Gamma(g)^{-1}\{0\} \cap \mathbb{D}$ to an order greater or equal than the order of vanishing of $\Gamma(g)$ at those points, and which vanish on $E$ (where the notion of "order of vanishing" makes no sense).

Notice that this allows us to compute precisely the codimension of $\mathcal{I}$, as the sum of the number of points in $E$, counted in the usual way, and the number of points in $E^{\prime}$, counted with multiplicities.

\section{ReFERENCES}

[1] R. G. Blumenthal, Holomorphically closed algebras of analytic functions, Math. Scan. 34 (1974), 84-90.

[2] J. L. Burchnall, T. W. Chaundy, Commutative ordinary differential operators, Proc. Roy. Soc. London, Ser. A 118 (1928), 557-583.

[3] M. J. Jaffrey, T. L. Lance, M. I. Stessin, Submodules of the Hardy space over polynomial algebras, Pacific J. Math., 194, No. 2 (2000), 373-392.

[4] J. Jones, Generators of the disk algebra, Ph.D. dissertation, Brown University, 1977.

[5] D. Khavinson, T. L. Lance, M. I. Stessin, Wandering property in the Hardy space., Michigan Math. J. 44 (1997), no. 3, 597-606.

[6] T. L. Lance, M. I. Stessin, Multiplication invariant subspaces of Hardy spaces, Canadian J. Math., 49 (1997), 100-118.

[7] A. I. Markushevich, Theory of functions of complex variables, Chelsea, 1977.

[8] M. B. Nathanson, Elementary methods in Number theory, Springer Verlag, Graduate Texts in Mathematics, 195 (2000).

[9] W. Rudin, The closed ideals in an algebra of analytic functions, Canad. J. Math. 9 (1957), 426-434.

[10] N. Sibony, J. Wermer, Generators for $A(\Omega)$, Trans. Amer. Math. Soc. 194 (1974), $103-114$.

[11] J. Wermer, Rings of analytic functions, Ann of Math. 67 (1958), 497-516.

[12] J. Wermer, Subalgebras of the disk algebra. Colloque d'Analyse Harmonique et Complexe, 7 pp. (not consecutively paged), Univ. Aix-Marseille I, Marseille, 1977. 
Michael I. Stessin

Department of Mathematics and Statistics University at Albany

Albany, NY 12222

USA

stessin@math.albany.edu
Pascal J. Thomas

Laboratoire Emile Picard, UMR CNRS 5580

118 route de Narbonne 31062 TOULOUSE CEDEX

France

pthomas@cict.fr 